\title{
Developments in Particle Detection
}

\section{Tejinder S. Virdee*}

Address

EP Division, CERN, Switzerland and Imperial College, London, UK. E-mail:

t. virdee@cern.chi

ABstract: From the many recent developments in particle detectors we select some of the major and challenging ones. The selected developments are primarily those necessitated by the harsh environment of the LHC. The presentations made in the parallel sessions of this conference are mostly placed in the context of these developments. This includes those connected with two new experiments concepts which were presented namely: TESLA and BTeV. From the inner tracking we look at pixel detectors, silicon microstrip detectors and microstrip gas detectors; from calorimetry we consider $\mathrm{PbWO}_{4}$ scintillating crystals and the 'accordion' liquid argon calorimeter; from the muon systems we consider monitored drift tubes (MDTs), thin-gap chambers (TGCs) and resistive plate chambers (RPCs). Another subject covered, and much reported in the parallel sessions, is that of photodetectors including avalanche photodiodes (APDs), vacuum phototriodes (VPTs), hybrid photodiodes (HPDs) and visible light photon detectors (VLPCs). Finally the important subject of electronics is discussed.

\section{Introduction}

A major R\&D effort in particle detectors was required during the last decade to select detectors that could survive and operate reliably in the harsh environment of the Large Hadron Collider (LHC). For each sub-detector technology retained, several others were also investigated. Development of new particle detectors takes a long time and goes through many phases starting from the idea or concept, progressing through intensive $\mathrm{R} \& \mathrm{D}$, prototyping, systems integration, installation and commissioning and finally data taking. This can be illustrated using any one of the many detector technologies in the LHC experiments.

We have chosen lead tungstate scintillating crystals, used for the electromagnetic

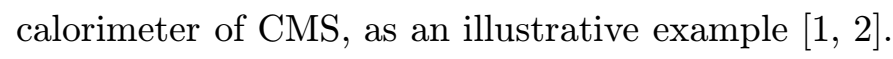

- Idea: in 1992 some yellowish samples of a few cc were shown - the final volume required is $10 \mathrm{~m}^{3}$ !!

\footnotetext{
${ }^{*}$ Speaker.
} 
- R\&D: 1993-2000: much work was carried out to increase the size of crystals (both length and cross-section), requiring improvements in the growing technique for heavier and heavier crystals. Improvements in the radiation hardness required optimization of stoechiometry (fraction of lead oxide and tungsten oxide), of the purity of raw materials (balance cost $\mathrm{v} / \mathrm{s}$ level of purity), and compensation of defects by specific doping (production crystals are now doped with Yttrium and Niobium).

- Prototyping: 1994-2000: performance of larger and larger matrices of crystals was studied in test beams - this should culminate in a test in 2002 of a prototype 'basket' comprising 400 crystals. The information gleaned from the earlier prototypes was fed back into the R\&D e.g. in 1997 significant radiation induced attenuation (through creation of colour centers) was observed at a low dose rate ( $1 \mathrm{~Gy} / \mathrm{hr})$ leading to a drop in the collected light. The extent of the loss depended on the dose rate and exhibited saturation. However after irradiation recovery of the light signal was observed. Through R\&D the light loss has been reduced by a factor of 5 at saturation: now it is $\sim 3-5 \%$.

- Mass manufacture: 2000-2006: a sizeable and high-yield crystal growing capability had to be put in place - around 130 ovens had to be refurbished and computer-control implemented. The diameter of the boules has been steadily increased so that now two crystals, and probably 4 crystals, can be obtained from one boule.

- Systems integration: 2001-2003: the crystals are assembled into the specially developed light and thin mechanical structures, the electronics, cooling system and signal and voltage cables have to be integrated into each one of 36 phi 'super-modules' comprising 4 'baskets' each.

- Test beam calibration (2002-2006)

- Data taking is foreseen in 2006.

It can be seen that almost one and half-decades will have passed from the concept to physics data taking!

\section{New Experiments}

Two new experiment designs were presented in the parallel session namely TESLA [3-i] and $\mathrm{BTeV}\left[\begin{array}{l}4 \\ 4\end{array}\right]$. It is clear that this new generation of experiments can benefit from the development of detectors for LHC and can therefore enter into the detector development cycles at later stages. The time from conception to completion can hence be shortened. We shall briefly describe these detectors in order to pull out novel detector technologies and place them in the context of the development of some of the LHC detectors.

\subsection{The TESLA Detector}

The parameters of the various electron-positron colliders are compared in Table 1. At TESLA the repetition rate is $5 \mathrm{~Hz}$ with 2820 bunches/train. The main requirements for 


\begin{tabular}{|c|c|c|c|}
\hline & TESLA & LEP & SLC \\
\hline Energy $(\mathrm{GeV})$ & 500 & 209 & 92 \\
\hline Luminosity $\left(\mathrm{cm}^{-2} \mathrm{~s}^{-1}\right)$ & $3.4 \times 10^{34}$ & $6 \times 10^{31}$ & $3 \times 10^{30}$ \\
\hline Bunch Spacing $(\mathrm{ns})$ & 337 & 22000 & $8.3 \times 10^{6}$ \\
\hline Beam size $(\mathrm{x}, \mathrm{y})(\mathrm{nm})$ & 553,5 & $3 \times 10^{5}, 8000$ & 1500,650 \\
\hline
\end{tabular}

Table 1: Some of the parameters of TESLA compared with those of LEP and SLC.

the TESLA detector are similar to those for the pp LHC experiments and are summarized below:

- Good momentum resolution of $\delta\left(1 / p_{T}\right)<5.10^{-5} \mathrm{GeV}^{-1}$ that is driven by the need for a precise measurement of recoil mass in processes such as $e^{+} e^{-} \rightarrow Z(\rightarrow \ell \ell) H$. The desired resolution is similar to that in ATLAS and CMS but a factor 10 better than that in LEP detectors.

- Vertexing - an impact parameter resolution of $\delta$ (impact parameter) $<5 \mu \mathrm{m}+10 \mu \mathrm{m}$ $/\left(\operatorname{psin}^{3 / 2} \theta\right)$. Such a resolution is necessary for identifying final states containing $\mathrm{c}$, $\mathrm{b}$ and $\mathrm{t}$ quarks in order to determine the branching ratios of $H \rightarrow b \bar{b}, c \bar{c}, t \bar{t}$. High purity and high efficiency tagging of $\mathrm{c}$ and $\mathrm{t}$ as well $\mathrm{as} \mathrm{b}$ is required.

- Energy flow - a large number of jets is expected in final states such as $Z H \rightarrow q \bar{q}$ $+b \bar{b}$ (i.e. $4 \mathrm{j}$ incl $2 \mathrm{~b}$ ), $t \bar{t} \rightarrow b W, b W$ (i.e. $6 \mathrm{j}$ incl $2 \mathrm{~b}), H A \rightarrow t \bar{t}+t \bar{t}(12 \mathrm{j}$ incl $4 \mathrm{~b})$. Beamstrahlung, initial state radiation and the complex topology of such final states makes kinematics fits difficult.

- Hermiticity - missing $E_{T}$ is an important signature for SUSY processes.

The single most important aspect of detector design is the choice of the magnet field configuration. In order to satisfy the above requirements a detector configuration similar to that of CMS has been chosen (Fig. 1); a large-bore 3-4 T solenoid enclosing the inner tracking and calorimetry with the muon placed system outside. The challenge associated with the design and construction of high field solenoids is considered in Section 3.

The inner tracking comprises a large volume TPC complemented by a multi-layer semiconductor vertex detector. The TPC provides a large number of measurement points for a minimum of material per point. It will be read-out continuously with data from about 160 bunch crossings overlaid during a drift time of $55 \mu \mathrm{s}$. During this interval $\sim 0.01$ highenergy hadronic event is expected. One readout technology being considered is the gas electron multiplier (GEM) coupled with microstrip gas chambers (MSGCs) (see Section 4.3). In GEM and MSGC detectors the problem of ion feedback is naturally reduced and GEM detectors lend themselves to gating that can further reduce ion feedback if required.

The vertex detector is designed to have a resolution of a factor two better than that achieved by SLD that used CCD technology: $\sigma(i p)_{r \phi}=4.2 \mu \mathrm{m} \otimes 4.0 \mu \mathrm{m} /\left(\operatorname{psin}^{3 / 2} \theta\right)$, where $\otimes$ signifies the root of the quadratic sum of the terms and $\mathrm{p}$ is in $\mathrm{GeV} / \mathrm{c}$. This is achieved by $20 \times 20 \mu \mathrm{m}^{2}$ pixels with a first layer at a radius of $1.5 \mathrm{~cm}$ from the beam-line. The 


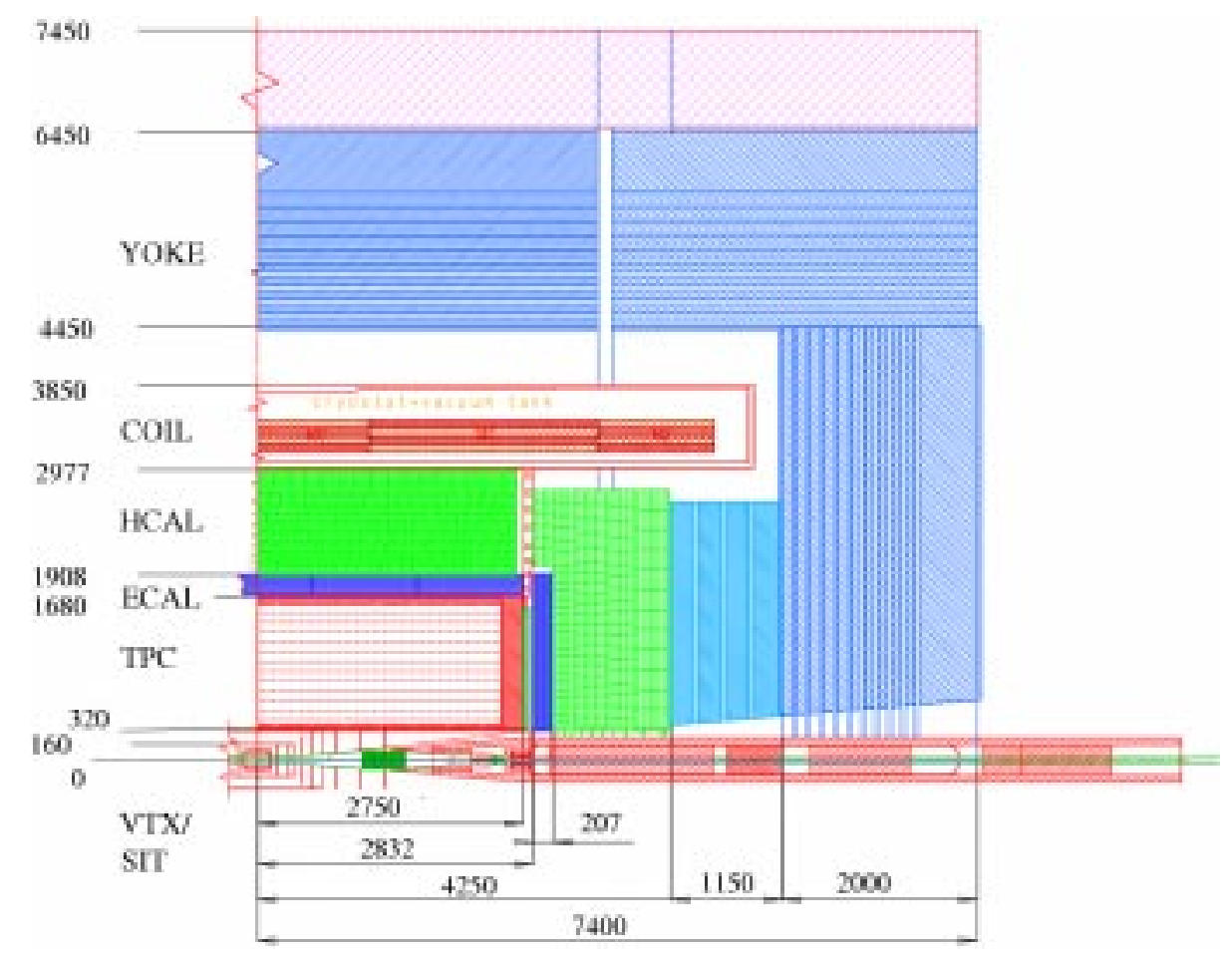

Figure 1: The transverse view of the TESLA detector. All dimensions are in mm.

expected hit rate in TESLA is $0.03 \mathrm{hits} / \mathrm{mm}^{2} /$ bunch crossing. Alternative technologies are CMOS monolithic pixels and hybrid pixels. All three approaches require considerable R\&D before use at TESLA. This is considered in Section 4.1.

Both the electromagnetic and hadronic calorimeters will be highly segmented to disentangle multi-jet events and enable use of energy flow algorithms. The aim is to improve the jet energy measurement by using the momenta of charged tracks measured in the inner tracking, the energies of photons and electrons measured in the ECAL and the energies of neutral hadrons measured in the hadron calorimeter. For optimal use of the energy-flow technique it is advisable to place the calorimetry inside the coil. Clearly the precision attained will depend on how the momenta and energies are combined, for which dense highly granular calorimeters are of great value. The electromagnetic calorimeter is planned to have a segmentation of $1 \times 1 \mathrm{~cm}^{2}$ using sampling of $1.4 \mathrm{~mm}$ tungsten/Si. The hadronic calorimeter uses $2 \mathrm{~cm} \mathrm{Fe} /$ scintillators with WLS fibre readout. The smallest tile size is $5 \times 5 \mathrm{~cm}^{2}$.

Since the bunch trains are $\sim 1 \mathrm{~ms}$ long, data have to be buffered before being read-out during the long inter-train interval of $200 \mathrm{~ms}$.

\section{$2.2 \mathrm{BTeV}$}

$\mathrm{BTeV}$ is designed to measure $\mathrm{CP}$ violation parameters in the b-quark system at the Tevatron. The special features are:

- a precision vertex detector comprising 31 pixel planes using hybrid pixels 
- particle identification assured by RICH detectors

- good measurement of mass of final states containing photons using a lead tungstate electromagnetic calorimeter.

\section{Superconducting Magnets}

The engineering challenges inherent in the superconducting magnets of ATLAS and CMS experiments tend to be overlooked. The parameters are compared in Table 2. The field generated in a solenoid is given by $\mathrm{B}=\mu_{0} \mathrm{nI}$. For CMS [ipin] with $\mathrm{B}=4 \mathrm{~T}, \mathrm{n}=2168$ implying that $\mathrm{I} \simeq 20 \mathrm{kA}$ thus requiring superconducting technology.

\begin{tabular}{|c|c|c|}
\hline & ATLAS Barrel Toroid & CMS Solenoid \\
\hline Field $(\mathrm{T})$ & 0.6 & 4.0 \\
\hline Inner Bore $(\mathrm{m})$ & $\mathrm{w}=5 \mathrm{~m}$ & $\phi=6$ \\
\hline Length $(\mathrm{m})$ & $\mathrm{L}=26 \mathrm{~m}$ & $\mathrm{~L}=13$ \\
\hline Current $(\mathrm{kA})$ & 20 & 19.5 \\
\hline Stored Energy $(\mathrm{GJ})$ & 3 & 2.7 \\
\hline Hoop Stress $(\mathrm{atm})$ & - & 64 \\
\hline
\end{tabular}

Table 2: Parameters of ATLAS and CMS superconducting magnets. and SLC.

Techniques developed for the construction of large solenoids for ALEPH and DELPHI experiements at LEP and H1 at HERA have been used in the design of the CMS solenoid. The main features that led to the high quality and reliability of these large magnets are the use of a high purity aluminium-stabilised conductor, indirect cooling (by thermosyphon) and full epoxy impregnation. However, a large increase in some parameters such as magnetic field, Ampere-turns, forces and stored energy (2.5 MJ) necessitated some changes. In particular a four-layer winding has been adopted using a novel conductor with a large cross-section $\left(22 \times 64 \mathrm{~mm}^{2}\right)$. The Rutherford cable is co-extruded with pure aluminium. This 'insert' is then electron-beam-welded, at the top and bottom of the insert, to two 'plates', made of an aluminium alloy, for the mechanical reinforcement.

In addition to understanding the basic conductor properties such as critical currents, mechanical and electrical properties of the cable and aluminium substrate it has been essential to establish, through $\mathrm{R} \& \mathrm{D}$ and pre-industrialization, the feasibility of industrial manufacture of the conductor and to identify the main factors driving its cost. Final fulllengths of the conductor are now being manufactured and the CMS magnet is scheduled for test in mid-2004.

\section{Inner Tracking}

The harshness of the conditions for inner tracking at the LHC can be illustrated by considering the radiation levels in the CMS tracking system (Table 3) for an integrated running time of 10 years corresponding to $5.10^{5} \mathrm{pb}^{-1}$. Three regions can be delineated. Pixel detectors are placed closest to the interaction vertex $(\mathrm{r}<20 \mathrm{~cm})$ where the particle flux is 
the highest. The typical size of a pixel is about $100 \mu \mathrm{m} \times 100 \mu \mathrm{m}$ leading to an occupancy of about $10^{-4}$ per LHC crossing. In the intermediate regions $(20<\mathrm{r}<60 \mathrm{~cm})$ the particle flux is low enough to enable use of Si microstrip detectors with typical cell size of $10 \mathrm{~cm} \times$ $50 \mu \mathrm{m}$ leading to an occupancy of $\simeq 1 \% /$ LHC crossing. In the outermost regions of the inner tracker $(\mathrm{r}>60 \mathrm{~cm})$ the particle flux has dropped sufficiently to allow use of gaseous (and of course Si microstrip) detectors. Typical cell sizes in this region are $25 \mathrm{~cm} \times 250$ $\mu \mathrm{m}$ giving an occupancy of a few percent per LHC crossing.

\begin{tabular}{|c|c|c|}
\hline Radius $(\mathrm{cm})$ & Fluence $\left(\times 10^{14} \mathrm{~cm}^{-2}\right)$ & Dose $(\mathrm{kGy})$ \\
\hline 4 & 30 & 800 \\
\hline 11 & 5 & 200 \\
\hline 22 & 1.5 & 80 \\
\hline 75 & 0.3 & 8 \\
\hline 115 & 0.1 & 2 \\
\hline
\end{tabular}

Table 3: The fluence of fast hadrons and dose expected over the first 10 years of LHC operation.

\subsection{Pixel Detectors}

At LHC and Linear Colliders (LC) many final states from new physics are expected to contain a substantial number of b-quarks and/or taus. The experimental aim is to reach a higher tagging efficiency and purity than has previously been achieved. For this the measuring layers have to be as close as possible to the beam-line, the point resolution (and two-track separation) has to be very good and the amount of material has to be kept to a minimum to minimize multiple scattering effects.

The LHC detectors have chosen hybrid pixel detectors comprising arrays of pixel sensors, of size $\sim 100 \times 100 \mu \mathrm{m}^{2}$, on a standard Si wafer, with electronics cells on a separate wafer. The electronics cells have the same footprint as the pixel sensors and are bumpbonded together. However the amount of material corresponds to (5-10) \% $\mathrm{X}_{0}$ per point. For this reason two other technologies are being pursued for TESLA: charge coupled devices (CCDs), and monolithic CMOS sensors.

\subsubsection{Charge Coupled Devices (CCDs)}

Since their invention in 1970 2-D CCDs have been used as electronic elements for storing and transferring charge in devices such as video cameras, telescopes and microscopes. CCDs found applications as particle detectors in mid-80s in the NA32 experiment [i6]. Subsequently they have been used in SLD in the VXD3 [īin]

In CCDs the signal charge is stored in potential pockets within a space-charge region. The charge is then moved from pocket to pocket towards the readout electrode by periodic changes of potential on gate electrodes underneath which are the pockets. The transfer is usually a slow process and hence such devices cannot be used at colliders such as the LHC.

However CCD-based detectors appear well adapted for use at linear colliders. The relevant features already present for SLD are: 
- small pixel sizes of $20 \times 20 \mu \mathrm{m}^{2}$, yielding an intrinsic spatial resolution of $\sim 4 \mu \mathrm{m}$ using charge sharing between pixels.

- very thin detectors (active thickness is $\sim 20 \mu \mathrm{m}$ ) leading to a small amount of material per point

However for TESLA a few features need improvement. The parameters that need improving can be seen from Table 4 . The major ones are:

- develop architectures to increase the speed of the pixel readout. VXD3 had a readout time of $180 \mathrm{~ms}$ ! The background, mainly from $\mathrm{e}^{+} \mathrm{e}^{-}$pairs, at $\mathrm{r}=1.5 \mathrm{~cm}$ is estimated to give $0.03 \mathrm{hits} / \mathrm{mm}^{2} / \mathrm{bx}$. Because of the TESLA beam structure the resulting $100 \mathrm{hits} / \mathrm{mm}^{2}$ (4\% occupancy) is not comfortable even at $50 \mathrm{MHz}$ readout speed.

- improvement of radiation hardness to a $1 \mathrm{MeV}$-equivalent neutron fluence of $10^{10}$ $\mathrm{n} / \mathrm{cm}^{2}$.

- develop thin mechanical structures to mount the CCDs

The active thickness of pixels is $20 \mu \mathrm{m}$. In order to keep the material at a minimum a method needs to be developed to thin down the pixels wafer and then to mount them on thin Be substrates.

Radiation damage could be an issue though the expected n-fluence is several orders of magnitude lower than that expected at the LHC. In

\begin{tabular}{|c|c|c|c|}
\hline & SLD & LC & Factor \\
\hline Longest CCD $(\mathrm{mm})$ & 80 & 125 & 1.6 \\
\hline Largest CCD area $\left(\mathrm{mm}^{2}\right)$ & 1280 & 3000 & 2.3 \\
\hline Ladder Thickness ( \% X0) & 0.4 & 0.12 & 3.3 \\
\hline Layer 1 radius $(\mathrm{mm})$ & 28 & 12 & 2.3 \\
\hline Readout rate $(\mathrm{MHz})$ & 5 & 50 & 10 \\
\hline N pixels $(\mathrm{M})$ & 307 & 700 & 2.3 \\
\hline
\end{tabular}

Table 4: The performance improvement factors required for LC CCD vertex detector. a sensitive thickness of $20 \mu \mathrm{m}$ the signal from a mip is only $\sim 2000$ e. This charge has to undergo hundreds of serial transfers before readout. In damaged detectors charge can be trapped in crystal defects. As crystal defects are especially abundant in the surface region, it is desirable to move the region of signal generation a certain distance away from the $\mathrm{Si}_{-} \mathrm{SiO}_{2}$ interface into the bulk. At the same time the speed of charge transfer also increases. This movement at a small distance from the surface is done in what is named a 'buried-channel' CCD. The charge is moved to an output electrode the size of which can be made very small, so that the detector capacity as seen by the readout electronics is very low (10-100 fF). However it is necessary to integrate at least part of the electronics onto the detector so that stray capacitances in leads $(\sim \mathrm{pF})$ can be avoided.

\subsubsection{Monolithic CMOS Pixel Sensors}

These are novel position sensitive devices that integrate a 2-D array of detecting diodes and the associated readout electronics on the same Si substrate [i, $]$. Hence the bump-bonding step, necessary for hybrid pixels, can be avoided. 
The architecture is similar to that of the visible light CMOS camera that is emerging as a competitor to the standard CCDs for digital photography. A thin epitaxial layer of low-resistivity silicon is used as the sensitive detector volume. The charge generated by the traversing charged particle ( $\sim 80$ electron-hole pairs per micron) is collected by an n-well/p-epi diode, created by n-well implantation into the epitaxial layer. The electrons liberated in this layer diffuse towards the diode within a typical time of a few tens of nanoseconds. Because of the three orders of magnitude between the doping levels of the p-epitaxial layer and of the neighbouring $\mathrm{p}^{++}$wells and substrate, potential barriers are created at the region boundaries, that act like mirrors for the excess electrons (Fig. 2).

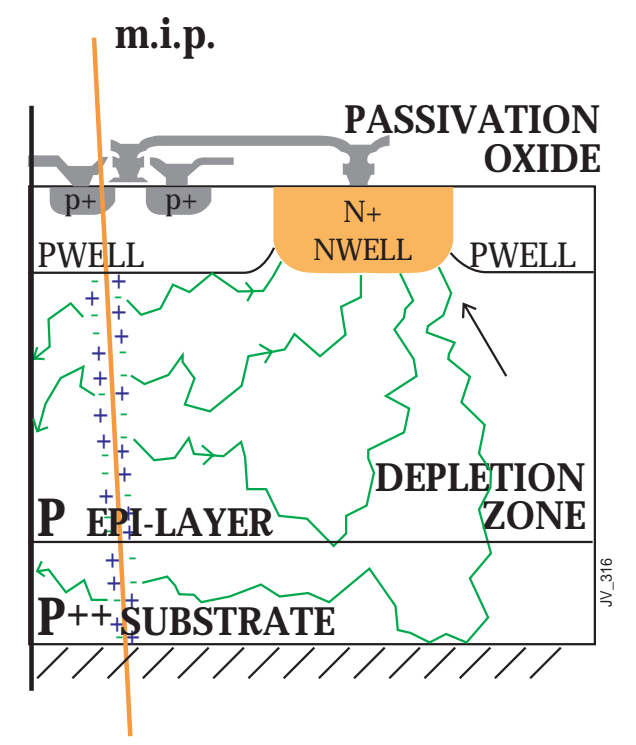

Figure 2: Charge collection within a CMOS pixel, indicating the potential barriers.

This principle was tested using $120 \mathrm{GeV} / \mathrm{c}$ pion beams on two prototype chips, each with $64 \times 64$ square pixels with almost a $100 \%$ fill-factor (active area), labeled MIMOSA I or II. The relevant characteristics and results are given in Table 5 [s్sis].

\begin{tabular}{|c|c|c|}
\hline & MIMOSA I & MIMOSA II \\
\hline Fabrication Process & $0.6 \mu \mathrm{m}$ & $0.35 \mu \mathrm{m}$ \\
\hline Epitaxial Layer Thickness & $14 \mu \mathrm{m}$ & $5 \mu \mathrm{m}$ \\
\hline Charge Collection Time & $150 \mathrm{~ns}$ & $100 \mathrm{~ns}$ \\
\hline Readout Clock Frequency & $5 \mathrm{MHz}$ & $25 \mathrm{MHz}$ \\
\hline Collected Charge (seed pixel) & $300 \mathrm{e}$ & $110 \mathrm{e}$ \\
\hline Collected Charge $(3 \times 3$ pixels $)$ & $900 \mathrm{e}$ & $315 \mathrm{e}$ \\
\hline Efficiency & $99.5 \%$ & $98.5 \%$ \\
\hline Spatial Resolution, $\sigma_{x}$ & $1.4 \mu \mathrm{m}$ & $2.2 \mu \mathrm{m}$ \\
\hline
\end{tabular}

Table 5: Characteristics and results from two CMOS monolithic pixel detectors.

As can be seen an excellent spatial resolution can be achieved with these very thin 
structures. However a $50 \%$ decrease in the collected charge was observed after proton irradiation with a fluence of $\sim 5 \times 10^{11} \mathrm{p} / \mathrm{cm}^{2}$. Further R\&D is needed to design faster read-out, demonstrate feasibility on full-scale chips $\left(\sim 2 \times 2 \mathrm{~cm}^{2}\right)$ and to improve radiation hardness. An important advantage of this approach is that standard sub-micron CMOS commercial fabrication processes can be used, which should lead to reduced cost and inherent radiation hardness, though the latter has still to be demonstrated. This is still an immature technique and may have a high potential.

\subsubsection{Hybrid Pixels.}

The LHC experiments employ hybrid pixel detectors close to the interaction region. The electronic cell contains amplification/shaping elements and circuitry to allow hit data to be stored on the edge of the chip awaiting readout upon receipt of a trigger.

An example of a hybrid pixel detector is the CMS pixels detector $[\overline{9}=1.96$. CMS has chosen $\mathrm{n}^{+}$pixels on $\mathrm{n}$-type Si substrate. The size of the pixels is $150 \mu \mathrm{m} \times 150 \mu \mathrm{m}$. The choice of substrate material is motivated by the large Lorentz angle $\left(34^{0}\right)$ for electrons in the $4 \mathrm{~T}$ field that leads to a sizeable charge sharing among the pixels. The spatial resolution in $\mathrm{r} \phi$ is $\sim 15 \mu \mathrm{m}$.

Results were presented from tests of BTeV prototype sensors bonded to $0.25 \mu \mathrm{m}$ 'deepsubmicron' electronics (see Section 8) [i $\left[\begin{array}{l}4 \\ 1\end{array}\right]$. The size of the pixels was $50 \times 400 \mu \mathrm{m}^{2}$. A r $\phi$ spatial resolution of $\sim 9 \mu \mathrm{m}$ was measured and the amplifier response was found not to change after irradiation up to a dose of 33 Mrad.

It appears unlikely that such a technique will be employed at the LC due to the large material thickness and large pixel sizes. However they afford a fast readout and exhibit high radiation resistance. Such a technique could well be suitable for upgrades of the closest-to-beam Si microstrip detectors at the LHC.

\subsection{Silicon Microstrip Detectors}

Silicon microstrip detectors are perhaps ideal detectors for inner tracking in the pp experiments at the LHC. They are fast (charge can be collected within an inter-bunch crossing time of $25 \mathrm{~ns})$, can give very good spatial resolution $(\sim 20-50 \mu \mathrm{m}$ leading to good momentum resolution) and a good two-track resolution (tracks inside high $\mathrm{p}_{T}$ jets can be separated). However in the early 1990's at the conceptual design stage of the LHC experiments, it was far from evident that large areas of Si microstrip detectors could be deployed. Radiation damage of detectors was poorly understood (hadron fluences of $10^{14} / \mathrm{cm}^{2}$ have to be withstood at the LHC), the costs of fabrication appeared prohibitive (100s of $\mathrm{m}^{2}$ are required) and the per channel electronics cost had to be brought down to $\sim$ a few $\mathrm{CHF} /$ channel (tens of millions of channels are required).

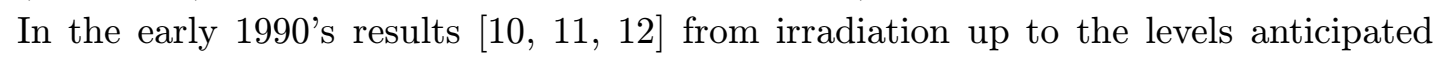
at the LHC showed a sizeable leakage current, that rises linearly with fluence, but more seriously that the effective doping of the bulk changed leading to type-inversion (n-type material becomes p-type). Progressively higher and higher bias voltage is then required ( $\sim$ several hundred volts) for full depletion. However, the available detectors could only be biased up to $\sim 100 \mathrm{~V}$ before exhibiting breakdown. It was also observed that the 
doping changes continued after the termination of irradiation for detectors kept at room temperature ('reverse annealing').

Considerable $\mathrm{R} \& \mathrm{D}$ has been carried out to improve the understanding of the damage mechanisms, working out strategies to prolong the useful lifetime of irradiated detectors and to improve the HV behaviour of the detectors.

Irradiation by hadrons creates defects in the Si lattice. These are interstitial defects Si atoms displaced to positions between regular lattice sites, or vacancies - empty lattice sites. These 'defects' are mobile at room temperature and if by chance an interstitial fills a vacancy 'annealing' is said to take place. There are other types of defects that are immobile at room temperature. These are: e-centres - a vacancy next to a phosphorous dopant atom that does not fulfil the role of donor anymore (donor removal), a-centres - vacancy-oxygen complex acting as an acceptor and a center for charge trapping, di-vacancy: two missing Si atoms right next to each other. These defects can capture electrons or holes that can be emitted after a delay and they can change the charge density in the space-charge region. Fig. 3 [i] 1 in] shows that starting with an n-type Si the doping first decreases with irradiation, the Si becomes intrinsic at a fluence of few time $10^{12} / \mathrm{cm}^{2}$, and the doping finally becomes effectively p-type and eventually rises linearly with fluence. This can be explained by removal of donors by creation of e-centres (vacancy-phosphorus defect) and creation of donor-like and acceptor-like defects. It can be parameterized as:

$$
N_{e f f}(\phi)=N_{e f f}(0) e^{-c \phi}-b \phi
$$

where $N_{\text {eff }}(0)$ is the initial effective concentration and $\phi$ is the fluence in $\mathrm{cm}^{-2}$. The constants $\mathrm{b}$ and $\mathrm{c}$ are found to be $7.9 \times 10^{-2} \mathrm{~cm}^{-1}$ and $3.5 \times 10^{-13} \mathrm{~cm}^{2}$ respectively. Since the bias voltage at full depletion is proportional to the effective doping the evolution of the bias voltage with fluence is shown in Fig. 4 [1] $\left.{ }_{1}^{1}\right]$. The plot also shows the evolution for low $(1-3 \mathrm{k} \Omega . \mathrm{cm})$ and high resistivity $(4-8 \mathrm{k} \Omega . \mathrm{cm})$ silicon. The hadron fluence at which type inversion occurs depends on the initial resistivity of the bulk material. In order to make sure that the depletion voltage can be kept low after long-term operation high (low) resistivity Si can be chosen for low (high) fluence regions (Fig. 4).

At the end of the irradiation the observed damage at room temperature diminishes with time ('annealing'), and so do the leakage current, the doping change and charge trapping. However after a certain period ( several weeks) the effective doping change starts to increase - an effect labeled 'reverse annealing'. Thus Si detectors run at room temperature in the LHC environment would sooner or later become unusable (depletion voltage would increase beyond breakdown voltage). Currently the only solution is essentially to keep $\mathrm{Si}$ detectors permanently at low temperatures, around $-10^{\circ} \mathrm{C}$, whether running or not in order to arrest reverse annealing.

Large leakage currents, up to several $\mu \mathrm{A}$ per strip, can be tolerated with fast amplifiers. With a current of $>5 \mu \mathrm{A}$ the power consumption of the detectors becomes comparable with that of the front-end electronics. However, since at the LHC the detectors have in any case to be run at low temperatures, the leakage current is much reduced (a factor of 2 reduction for a decrease of $\sim 7^{0} \mathrm{C}$ ). 


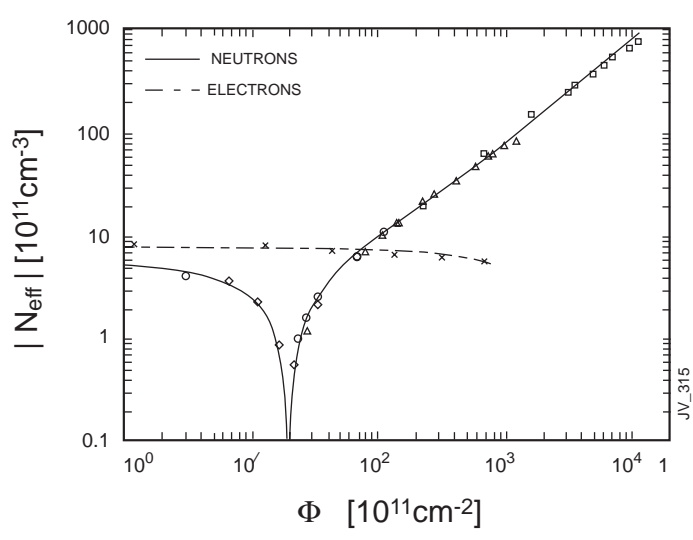

Figure 3: Effective impurity concentration versus fluence. The solid curve represents a fit to equation (4.1)

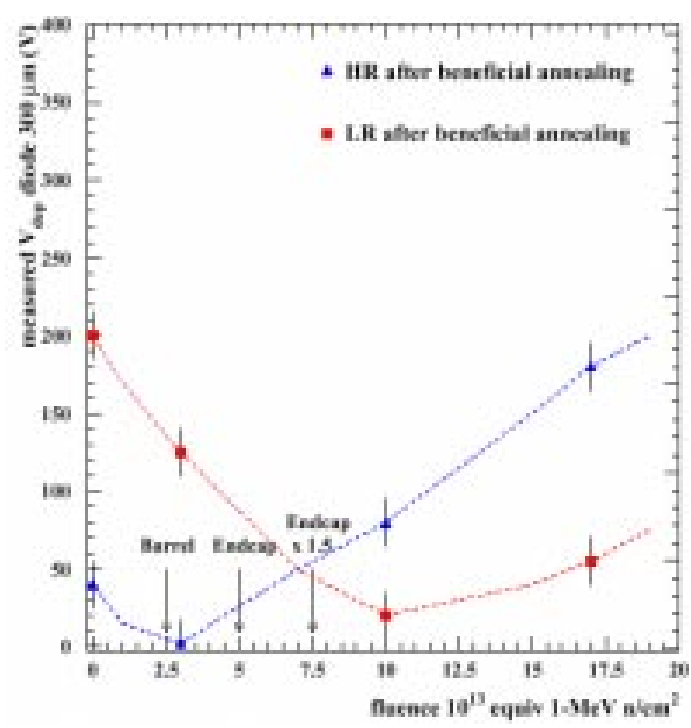

Figure 4: Measured depletion voltage for 300 $\mu \mathrm{m}$ thick Si diodes. HR - high resistivity 3-8 $\mathrm{k} \Omega \mathrm{cm}, \mathrm{LR}$ - low resisitivity $1-3 \mathrm{k} \Omega \mathrm{cm}$

Microstrip detectors formed using $\mathrm{p}^{+}$implantation on the front side to define strips on an n-type substrate (and a back side $\mathrm{n}^{+}$implantation for ohmic contact) are cheaper to fabricate; double sided processing can be avoided. However, as considered above, high bias voltages $(\sim 500 \mathrm{~V})$ have to be foreseen after type inversion. In the early 1990's operation at such voltages was considered to be difficult. With careful processing and use of multiple guard rings the field strengths at the implant edges have been considerably reduced. It is now usual to produce detectors that can withstand very high bias voltages.

It was further discovered that for high resistivity material the interstrip capacitance did not alter after irradiation for $<100>$ material; whereas for $<111>$ material it increased by $\sim 40 \%$ leading to worsened $\mathrm{S} / \mathrm{N}[1] \overline{1} \overline{3}]$. It is presumed that in $<111>$ material a large number of bonds are left 'dangling' at the surface that can act as traps for mobile charges.

Driven by micro-electronics technology, that requires larger and large diameter Si ingots, the cost per unit area of simple microstrip Si detectors fabricated using 6" technology has substantially decreased making the use of large areas affordable.

All of these developments led CMS to move to an all-Si inner tracker in 1999 [1] surface area of $\sim 225 \mathrm{~m}^{2}$ of p-on-n Si microstrip detectors, comprising $\sim 10 \mathrm{M}$ electronics channels, is envisaged: ATLAS has also chosen the p-on-n technology. Apart from cost considerations such a large area could be contemplated because a large degree of automation has been introduced in 'module' production, again benefiting from techniques employed in the micro-electronics industry. A typical CMS module comprises two $10 \times 10 \mathrm{~cm}^{2}$ silicon detectors, suitably aligned and glued together, with their strips bonded, and with one end 


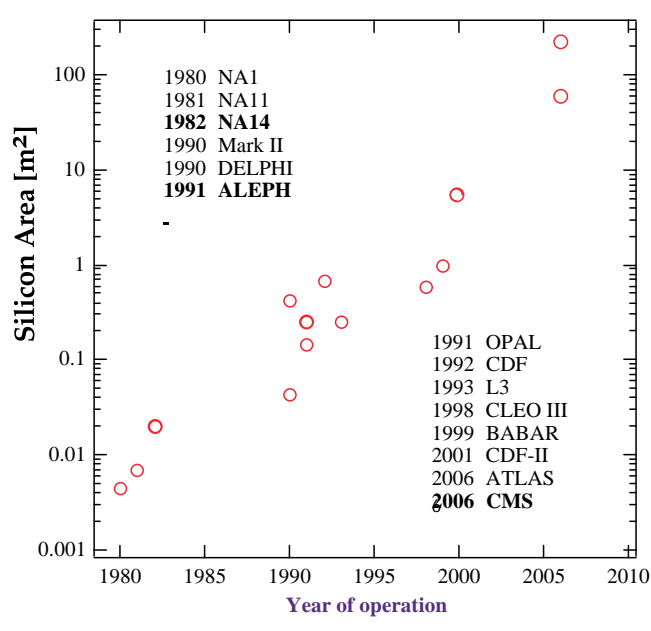

Figure 5: Evolution of the Si surface area used in various experiments. The year of startup is indicated.

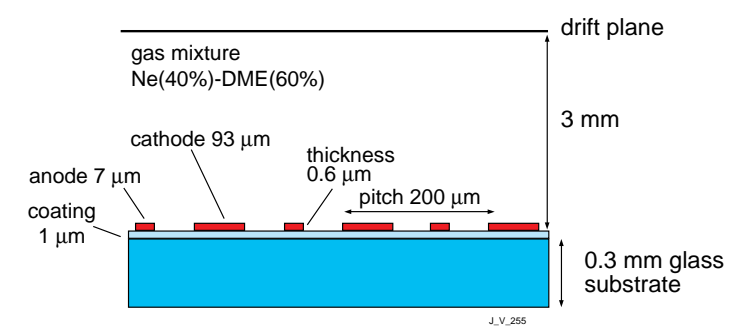

Figure 6: The layout of a microstrip gas chamber.

of the strips bonded to front-end electronics.

LHCb has chosen n-on-n detectors after irradiation tests of both types of detectors [1] tracking efficiency can remain high even with depletion thickness down to $60 \%$. Since the area involved is small, cost is not as important an issue as for ATLAS and CMS.

Nowadays in addition to the properties discussed above standard 'radiation resistant' Si microstrip detectors are considered to have the following characteristics: AC coupling, polysilicon bias, standard $0.20-0.25$ width/pitch ratio and metal overhang on the strips [i] $\left.{ }_{1}^{1} \overline{3} \overline{1}\right]$.

Interesting lessons can be learnt from the Si-microstrip detector upgrades for CDF and D0. Use of simple detector geometries and procedures assure good electrical and mechanical quality - single sided detectors are much easier to assemble than double-sided ones. For design and assembly it is desirable to have hybrids located off the silicon wafers and outside the tracking volume if possible. For mass assembly the timely availability of parts is essential. The front-end electronics should be robust and simple to manufacture. Many problems arose from delays encountered in the delivery of electronic chips from the foundries and failures induced by dicing of chips etc. An overriding message is to test mass production as early as possible.

In the space of 20 years silicon microstrip detectors have grown from covering areas of tens of $\mathrm{cm}^{2}$ to the hundreds of $\mathrm{m}^{2}$ now being manufactured (Fig. 5) [i $\left.1 \overline{1}^{2}\right]$.

$\mathrm{R} \& \mathrm{D}$ on Si microstrip and pixel detectors is continuing on for example macro-pixel detectors, defect engineered silicon, detectors with novel biasing schemes, detectors run at 
cryogenic temperatures etc.

\subsection{Microstrip Gas Chambers (MSGCs)}

MSGCs are made using micro-electronics technology where the precision of photo-lithography is $\simeq 0.1-0.2 \mu \mathrm{m}\left[i \overline{1} \overline{\hat{b}_{1}}\right]$. This overcomes two major limitations of multi-wire proportional chambers (MWPCs). In MWPCs the spatial resolution orthogonal to the wire is limited by wire spacing. The limit is around $1 \mathrm{~mm}$ due to mechanical and electrostatics considerations. The rate capability of MWPCs is limited by the long ion collection time that is typically several tens of $\mu$ s.

The MSGC is a miniaturized version of a MWPC (Fig 6). The wires are replaced by thin strips imprinted on an insulating support e.g. glass to prevent the electrostatic forces from distorting or breaking them. Hence their spacing and width can be reduced. The cathode and anode strips are laid on the substrate over which is placed a planar electrode generating the drift field. The drift distance of ions is therefore only $\sim 50 \mu \mathrm{m}$ compared with mm's in the case of MWPCs. MSGCs have been operated at rates up to $10^{6}$ particles $/ \mathrm{mm}^{2} / \mathrm{s}$. The gas gap between the substrate and the drift electrode is usually about $3 \mathrm{~mm}$.

The performance of MSGCs depends strongly on several critical parameters. The influence, and the choice of the value, of these parameters has been the subject of much $\mathrm{R} \& \mathrm{D}$ in the last decade. Much of this $\mathrm{R} \& \mathrm{D}$ has been driven by the desire to use these detectors in experiments such as HERA-B and CMS where prolonged running at high particle intensities is required. This desire also led to the invention of the Gas Electron

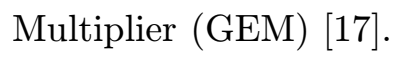

Coating the surface of the glass with resistive coating (e.g. diamond-like carbon coating or sputtering $1 \mu \mathrm{m}$ of electronic conducting Pestov glass with $\rho \simeq 10^{16} \Omega / \mathrm{cm}^{2}$ stops charging-up of the substrate at high rates and renders the detector independent of the bulk electrical characteristics of the substrate.

Strips made out of gold slow ageing and reduce attenuation of the collected charge along long strips. Early designs used aluminium as strip material and led to rapid ageing as aluminium is an active metal that can react vigorously with substances produced in the avalanche. Chromium yielded good results but has a relatively high specific resistance and hence is unsuitable for detectors with long strips.

Certain regions of the MSGC, e.g. those close to the electrode structures, have higher than average electric fields that may induce formation of streamers or sparks. It was found that for MSGCs exposed to heavily ionising tracks at high rates these sparks could damage the detectors such that the maximum voltage that can be applied before breakdown is reduced below the operating voltage. Two solutions have been pursued. The first one is to passivate the cathode edges by laying $8 \mu \mathrm{m}$ wide and $2 \mu \mathrm{m}$ thick strips of polyamide on the cathode edges. In a recent development, the Gas Electron Multiplier (GEM), the gain is achieved in two stages.

To demonstrate the suitability of MSGCs for CMS, with passivation of cathodes [i] $\overline{1}_{1} \overline{1}$, around 30 chambers were subjected to $350 \mathrm{MeV}$ pions at an intensity of $\sim 6 \mathrm{kHz} / \mathrm{mm}^{2}$ for a total integrated time of around 500 hours. The chambers had a size of $20 \times 10 \mathrm{~cm}^{2}$ and 
comprised a total of over 16k strips. These chambers were assembled in industry following relatively strict QC procedures. The operating voltage $(\sim 500 \mathrm{~V})$ was set individually for each chamber to attain an efficiency of $98 \%$ for a mip. The gas mixture used in 'passivated chambers' is Neon-DME (40 \%-60 \%). DME has large primary cluster and total ionization density $(60 / \mathrm{cm})$. In the test very few strips were found to have been damaged and the spark rate was found to be less than 1 spark/chamber/day at the end of the test period. It was clearly demonstrated that such chambers could withstand the LHC environment and operate well.

In GEM the first stage of amplification is carried out in a thin polymer foil (kapton), metal-clad on both sides and perforated with a high density of holes by photo-lithographic processing. When a suitable potential difference is applied between the two sides, the field in the hole is large enough to allow multiplication. A simulation of the multiplication process can be seen in Fig 7 [i] $\left.\underline{1}_{1}\right]$. The second stage can be a conventional MSGC (Fig. 8). The MSGC can then be operated at a lower voltage thus promising a safe operation over a wide range of voltages. In GEM detectors $\mathrm{Ar} / \mathrm{CO}_{2}$ is usually used.

Results were reported from a systematic study of the operating parameters of MSGC+GEM detectors [1] $\left[\begin{array}{l}1 \\ \underline{1}\end{array}\right]$. The transparency of the GEM mesh can be kept close to $100 \%$ for a drift

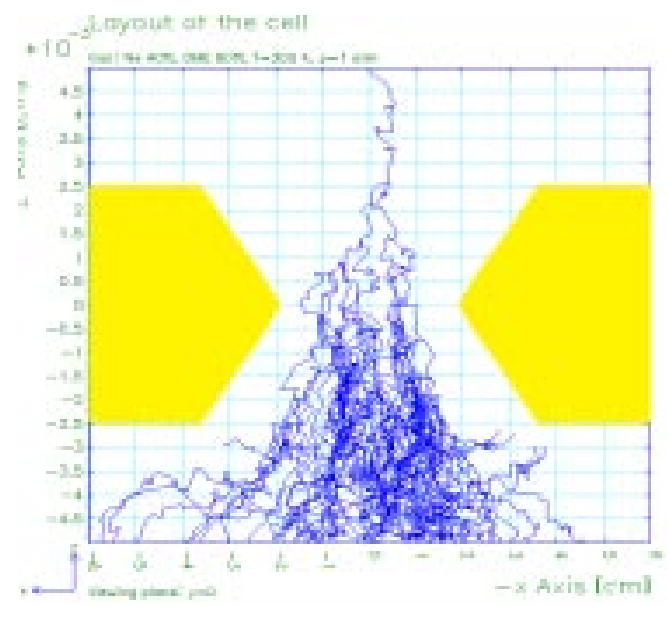

Figure 7: Avalanche multiplication in a GEM

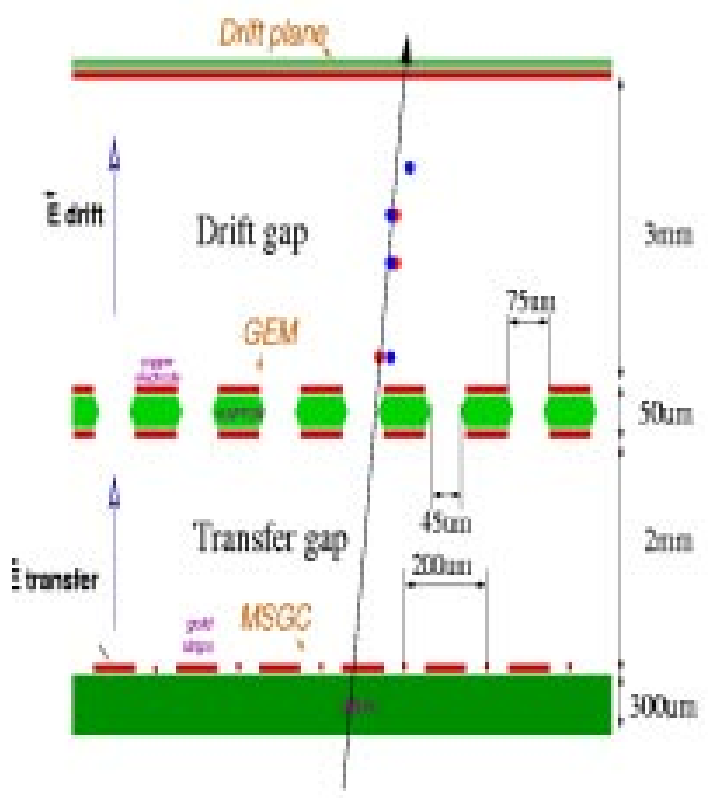

Figure 8: Layout of a GEM+MSGC chamber

field between 0.5 to $3.0 \mathrm{kV} / \mathrm{cm}$, a transfer field of $3.5 \mathrm{kV} / \mathrm{cm}$ and GEM potential difference of $400 \mathrm{~V}$. The situation was simulated showing that under these conditions all the field lines from the drift region enter the GEM holes.

Results were presented from 184 MSGC + GEM chambers constructed for the HERA- 
B experiment [20 $\left.{ }_{2}^{2}\right]$. The chambers have a size of $30 \times 30 \mathrm{~cm}^{2}$. The MSGC substrate was coated with amorphous carbon, the width of the anodes and cathodes was $10 \mu \mathrm{m}$ and 170 $\mu \mathrm{m}$ respectively at a pitch of $300 \mu \mathrm{m}$. The GEM holes have a pitch of $140 \mu \mathrm{m}$ and a diameter of $90 \mu \mathrm{m}$. The lessons learnt from the long development phase were:

- tests have to be carried out in high intensity hadron beams

- irradiation should be carried out over as large an area as possible

- materials and gas mixtures should be chosen very carefully to minimize ageing

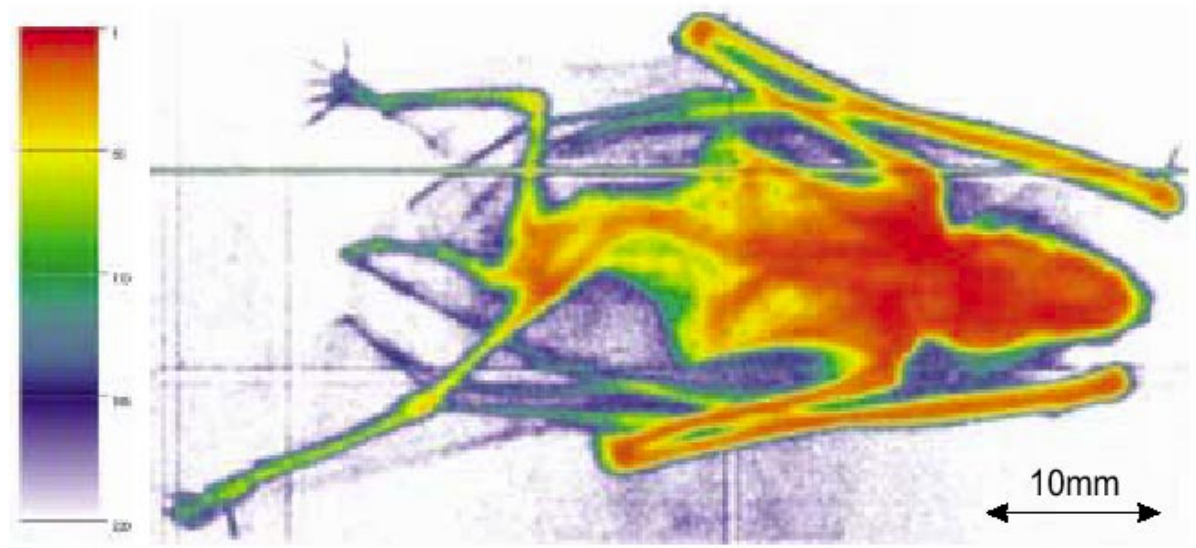

Figure 9: X-ray absorption image of a small mammal taken with a double-GEM detector.

The detectors needed conditioning under stable beam. One GEM spark/chamber/day was observed though 3 chambers had frequent GEM sparks. The voltages of the chambers had to be individually set as there was a large variation of gain.

MSGC+GEM chambers are being considered for the readout of the TPC in TESLA.

Much research is still being carried out on these miniaturized gas chambers. The introduction of GEM means that the effective gain can be adapted to particular needs by having one or more stages of GEM amplification. The big advantage is that the gas amplification and read-out stages can be separated. As the signal is induced by the motion of electrons, the probability of discharge is almost eliminated. As an example, Fig. 9 shows a radiographic image of a bat taken using a chamber comprising a double GEM with 2-D

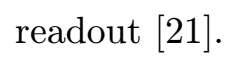

\section{Calorimetry}

Considerable research was carried out to select and then develop the electromagnetic calorimeters of the LHC pp experiments. ATLAS chose liquid argon sampling calorimeter for the electromagnetic calorimeter and the endcap hadronic calorimeter. CMS chose lead tungstate scintillating $\left(\mathrm{PbWO}_{4}\right)$ crystals for its electromagnetic calorimeter. Both experiments use scintillator based hadronic calorimeters with wave-length shifting fibres. 


\subsection{ATLAS Accordion Liquid Argon Sampling Electromagnetic Calorimeter}

Conventionally the absorber planes in ionization chambers are oriented perpendicular to the incident particles. However in geometry it is difficult to

- realise fine lateral segmentation with small size towers, which in addition need to be projective in collider experiments,

- implement longitudinal sampling,

without introducing insensitive regions, a large number of penetrating interconnections, and long cables which necessarily introduce electronics noise and lead to increased charge transfer time. To overcome these shortcomings a novel absorber-electrode configuration, known as the 'accordion' (Fig. 10, [i2 2i $]$ ), has been introduced, in which the particles traverse the chambers at angles around $45^{\circ}$. With such structures the electrodes can easily be grouped into towers at the front or at the rear of the calorimeters.

In ATLAS ECAL the absorber is made of lead plates, clad with thin stainless steel sheets for structural stiffness and corrugated to the shape shown in Fig. 11. Details of the sampling structure can also be seen. The read-out electrodes are made out of copper clad kapton flexible foil and kept apart from the lead plates by a honeycomb structure.

Results from a full-size barrel prototype module were presented. The final functionality electronic readout chain was employed but not in the radiation-hard version. The local energy resolution for electrons could be parametrized by

$$
\sigma(E) / E=(9.18 \pm 0.13) \% / \sqrt{E} \oplus(0.27 \pm 0.04) \%
$$

where $\mathrm{E}$ is in $\mathrm{GeV}$. The $\mathrm{e} / \mu$ ratio is measured to be $0.75 \pm 0.03$. The photon-pointing res-

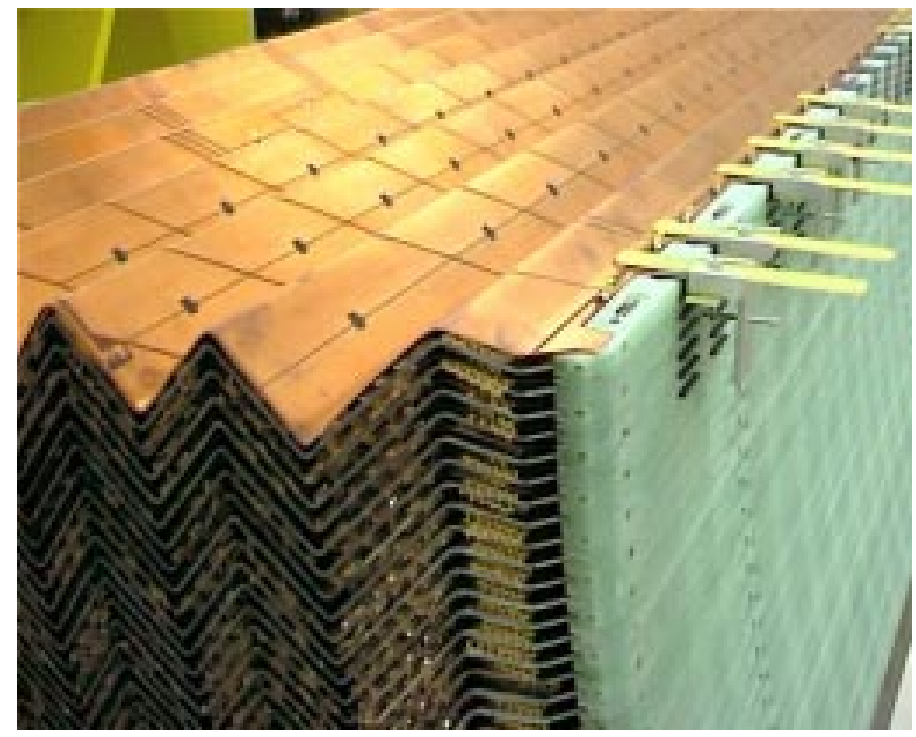

Figure 10: A view of the ATLAS Barrel Module 0 liquid argon "accordeon" calorimeter wedge

olution, necessary to measure direction of photons from the $H \rightarrow \gamma \gamma$ decay, was measured to satisfy the requirement of $50 \mathrm{mrad} / \sqrt{E}$. 


\subsection{ATLAS Endcap Liquid Argon Hadronic Calorimeter}

The ATLAS endcap hadronic calorimeter has a conventional structure but for a novel readout that employs 3 readout electrodes/gap linked serially, in an approach termed 'electrostatic transformer structure'. Copper plates, with a thickness of $25 \mathrm{~mm}$ and $50 \mathrm{~mm}$, sandwich argon gaps of a width of $\sim 8 \mathrm{~mm}$. Several modules of HEC were tested [i2 $2 \overline{2}]$. The energy resolution could be parametrized by

$$
\sigma(E) / E=(70.6 \pm 1.5) \% / \sqrt{E} \oplus(5.8 \pm 2.2) \%
$$

The $\mathrm{e} / \mu$ ratio is measured to be $0.93 \pm 0.04$. The $\mathrm{e} / \mathrm{h}$ ratio was measured to be 1.5 \pm 0.02 . The series production of both the electromagnetic and hadronic modules is well underway.

\section{Photodetectors}

The parallel session had many presentations on developments of photodetectors.

\subsection{Silicon Avalanche Photodiodes and Vacuum Phototriodes}

The electromagnetic calorimeter for CMS uses lead tungstate $\left(\mathrm{PbWO}_{4}\right)$ scintillating crystals as the active medium. In many respects $\mathrm{PbWO}_{4}$ is an ideal crystal for use at the LHC. It has:

- a very short radiation length $(8.9 \mathrm{~mm})$ and Moliere radius $(21.9 \mathrm{~mm})$ allowing a compact design

- fast scintillation emission (>90\% of the light emitted within $100 \mathrm{~ns}$ ) in the visible region $(\sim 430 \mathrm{~nm})$

- strong resistance to radiation induced darkening

However the light yield is rather low $(\sim 50$ photons $/ \mathrm{MeV})$ necessitating the use of photodetectors with internal gain. Furthermore, in CMS the ECAL is located in a solenoidal magnetic field of $4 \mathrm{~T}$. Therefore photodevices that can provide some gain and operate in a magnetic field are required. The photodetectors chosen in the barrel are avalanche photodiodes $[\overline{2} \overline{3}]$. In the endcap the radiation levels are much higher than in the barrel and the use of silicon based photodetectors such as APDs is excluded: vacuum phototriodes are used in this region [2] $2 \overline{4}$. The integrated radiation levels over 10 years of LHC operation in the barrel part of CMS are $2.10^{13} \mathrm{n} / \mathrm{cm}^{2}$ and $2.5 \mathrm{kGy}$ whilst in the endcap region, and $|\eta|=2.6$, the levels are $10^{14} \mathrm{n} / \mathrm{cm}^{2}$ and $25 \mathrm{kGy}$.

\subsubsection{Avalanche Photodiodes (APDs)}

APDs are novel devices; only a handful were available in the early 1990's. CMS will use 120,000 devices (2 per crystal) [20표. CMS has developed these APDs over 8 years in close collaboration with Hamamatsu. The structure of these APDs is shown in Fig. 11. Incident photons produce photoelectrons in the surface layers $(\sim 5 \mu \mathrm{m}$ thick $)$ that are accelerated 
in the high field region of the p-n junction. They gain enough energy to create further electron-hole pairs and an avalanche is generated. The diode structure is embedded in $\sim 40 \mu \mathrm{m}$ of eptaxially grown silicon on a low resitivity silicon base. The grooves etched around the edges reduce surface currents and are important for radiation hardness. Some important characteristics of APDs are listed in Table 6.

\begin{tabular}{|c|c|}
\hline Active area (2 APDs/crystal) & $5 \times 5 \mathrm{~mm}($ each $)$ \\
\hline Quantum efficiency & $75 \%$ at $430 \mathrm{~nm}$ \\
\hline Operating voltage & $\sim 380 \mathrm{~V}$ \\
\hline Gain $(\mathrm{M})$ & $50(\max >1000)$ \\
\hline Capacitance & $80 \mathrm{pF}$ \\
\hline Voltage sensitivity $(1 / \mathrm{M} \times \mathrm{dM} / \mathrm{dV})$ & $3.15 \% / \mathrm{V}$ \\
\hline Temperature sensitivity $(1 / \mathrm{M} \times \mathrm{dM} / \mathrm{dT})$ & $-2.2 \% /{ }^{0} \mathrm{C}$ \\
\hline Excess noise factor & 2.1 \\
\hline Thickness sensitive to ionizing particles & $5 \mu \mathrm{m}$ \\
\hline
\end{tabular}

Table 6: Some important characteristics of APDs.

Amongst the improvements made in the course of this development are:

- the amplification region has been placed just behind the conversion layer to substantially decrease the sensitivity to traversing charged particles.

- the epitaxial layer is grown on a few hundred microns thick low resistivity silicon to lower the capacitance

- the tolerance to radiation has been improved after changing the geometry to reduce lateral electric field, by rounding corners, changing spacings and introducing field clamps.

Radiation hardness proved to be hard to assure. An important indicator of radiation hardness was found to be the difference between the breakdown $\left(\mathrm{V}_{B}\right)$ and operating voltages $\left(\mathrm{V}_{C}\right)$ (the difference $\left(\mathrm{V}_{B^{-}}-\mathrm{V}_{C}\right)$ for good APDs is $\sim 40 \mathrm{~V}$ ).

Since the aim is to reach a failure rate of only $0.1 \%$ over 10 years of LHC operation all APDs will be screened by non-destructive irradiation at $5 \mathrm{kGy}$ with ${ }^{60} \mathrm{Co}$. This will be followed by 2 weeks of annealing and accelerated ageing at $90^{\circ} \mathrm{C}$ to remove all the dark current induced by the irradiation. Any APD showing a large $\mathrm{V}_{B}-\mathrm{V}_{C}$ or an anomalously large leakage current will be rejected. Destructive tests with neutron irradiation have also been carried out. Some 500 screened APDs have been irradiated without any failure.

Around twenty thousand APDs have already been delivered to CMS.

\subsubsection{Vacuum Phototriodes (VPTs)}

A vacuum phototriode is essentially a photomultiplier tube with a single stage of gain (Fig. 12 [20, $\left.\left.\overline{2}_{1}\right]\right)$. After emission from the photcathode the photolelectrons are accelerated towards the anode consisting of a very fine metal mesh. A fraction $(\sim 50 \%)$ of the photons pass 


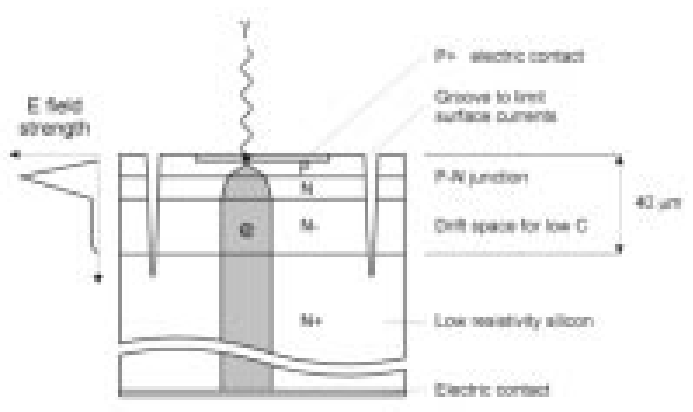

Figure 11: Schematic of an APD

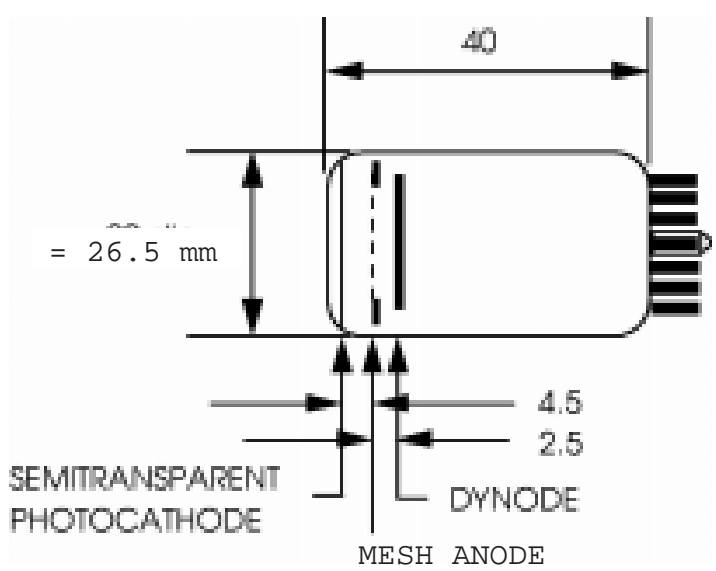

Figure 12: Schematic of a VPT

through the anode and strike a solid metal dynode, located behind the anode. Secondary electrons ejected from the dynode are accelerated back towards the anode where most are collected. Under normal operating conditions $\left(\mathrm{V}_{A}=1000 \mathrm{~V}, \mathrm{~V}_{D}=800 \mathrm{~V}\right)$ the effective gain of the VPT is $\sim 10$.

In CMS, the angle between the axis of the VPT and the direction of the magnetic field will vary from $\sim 6^{0}$ to $26^{0}$. It has been verified that the gain of the VPT does not vary by more than $10 \%$ in the range of angles from $-30^{0}$ to $+30^{0}$.

Radiation hardness is an issue because radiation can cause darkening of the window due to the formation of colour centers. Tubes with a standard borosilicate window are readily darkened by radiation. Tubes with fused silica windows are tolerant but would be prohibitively expensive for application in CMS. The use of UV transmitting glass is a good compromise that shows a small loss of transmission $(\sim 4 \%)$ at the doses mentioned above.

\subsubsection{Visible Light Photon Counter (VLPC)}

A novel item of the D0 upgrade is the scintillating fibre tracker employing 77,000 fibres, with a diameter of $835-\mu \mathrm{m}$, arranged in axial and $\mathrm{u}, \mathrm{v}$ steros doublets. The light is detected by a solid state device developed by Rockwell called the visible light photon counter (VLPC)

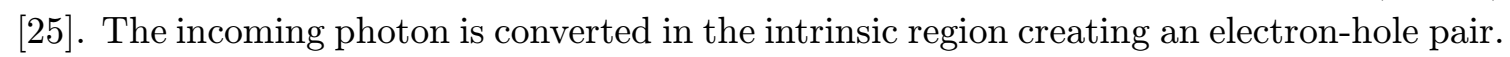
The hole drifts into the drift region where it ionizes an atom. The electron is accelerated back through the gain region generating avalanche amplification. Gains of 20,000 to 60,000 can be attained. These devices have high quantum efficiency ( $80 \%$ in the visible). The single photon response is excellent. An inconvenience of these devices is the need to run at $7 \mathrm{~K}$. In D0 each scintillating fibre is connected to a 7-11 $\mathrm{m}$ long clear fibre that transmits the light to the VLPCs kept in a dewar outside the experiment.

\subsubsection{Hybrid Photodiodes (HPDs)}

HPDs are two stage vacuum devices: photons are converted in a conventional photocathode 
and the photoelectrons are accelerated in an electric field of $10 \mathrm{kV} / \mathrm{cm}$. The energetic photoelectrons then strike a silicon detector that acts as an anode. Electron-hole pairs are created (one per $3.7 \mathrm{eV}$ of deposited energy) resulting in a gain of the order of 3000 .

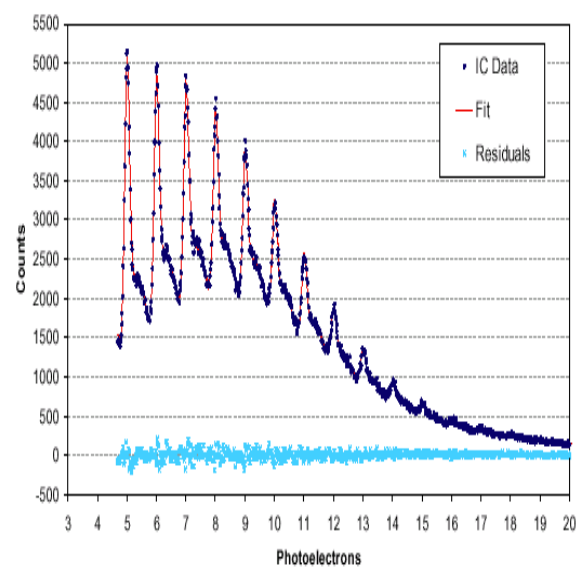

Figure 13: The response of an HPD

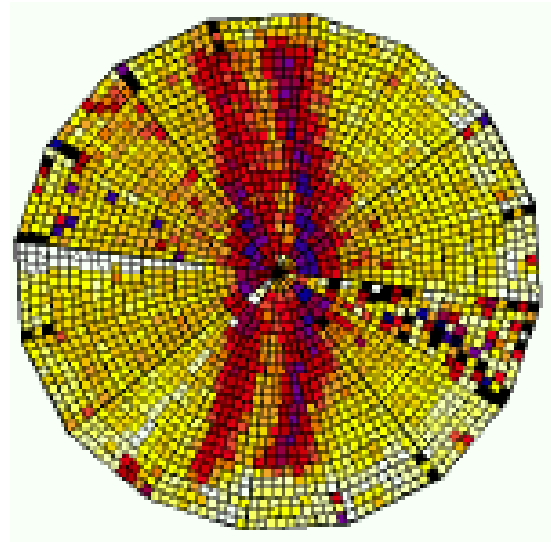

Figure 14: The separation of proton and pion rings produced by a beam of $8 \mathrm{GeV} / \mathrm{c}$.

These photodevices can operate in axial magnetic fields. The single photoelectron response of such devices is excellent as can be seen from Fig. $13[\overline{2} \overline{2} \overline{6}]$. At LHC they are used to detect light from the scintillators of the CMS HCAL and to detect Cerenkov photons from the LHCb RICH [i] $\left.\bar{z}_{1}\right]$.

CMS uses commercially manufactured 19 pixel channel devices in a standard 2' envelope. Results were presented in this conference from tests of specially prepared HPDs, used

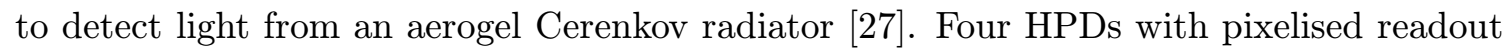
consisting of 2048 channels were used. The fountain shaped electric field gives a 2.4 fold de-magnifed image. The separation of proton / pion rings produced by a beam of $8 \mathrm{GeV} / \mathrm{c}$ is shown in Fig. 14.

\section{Muon Chambers}

Perhaps the biggest challenge for the measurement of momentum and identification of muons in the LHC pp experiments is the manufacture of thousands of $\mathrm{m}^{2}$ of chambers with the desired high precision (as low as $80 \mu \mathrm{m}$ per measured point) and including alignment procedures able to maintain such accuracy over large surface areas. These chambers use technologies that are not necessarily novel. However, much R\&D was required to demonstrate the required rate capability and good long-term operation under the modest levels of radiation anticipated in these regions.

For precision measurements both ATLAS and CMS use drift devices in the barrel region (Drift Tubes (DTs) and Monitored Drift Tubes (MDTs) respectively) and Thin 
Gap Chambers (TGCs) and/or Cathode Strip Chambers (CSCs) in the endcap regions. Both experiments use Resistive Plate Chambers (RPCs) for precise timing and triggering. CMS also uses the precision chambers for triggering.

Results were presented on measurements from the ATLAS muon chambers and their production status [2] $[\overline{8} \overline{-}]$.

\subsection{The Monitored Drift Tubes (MDTs)}

The MDTs are cylindrical aluminium tubes with a length of about $5 \mathrm{~m}$, a diameter of 3 $\mathrm{cm}$ and a wall thickness of $400 \mu \mathrm{m}$ with a central $50 \mu \mathrm{m}$ diameter W-Re wire. The tubes are operated with a non-inflammable $\mathrm{Ar}-\mathrm{CO}_{2}(93 \%-7 \%)$ gas mixture at a pressure of 3 bars. The wire is set at a potential of $\simeq 3100 \mathrm{~V}$ and the electric field at the wire is $\simeq$ $200 \mathrm{kV} / \mathrm{cm}$ yielding a low gain of about 20,000 to minimize ageing. The maximum drift time is $\simeq 700 \mathrm{~ns}$. MDTs are constructed from $2 \times 4$ monolayers of drift tubes for the inner and $2 \times 3$ mono-layers for the middle and outer stations. The structural components are 3 'cross plates' to which tube multi-layers are attached, and two 'long beams' connecting the cross plates. On the cross plates, an in-plane optical alignment system is mounted aiming at measuring wire displacements to an accuracy of $10 \mu \mathrm{m}$. Nearly 40,000 drift tubes have been produced corresponding to 80 chambers. Around 10 chambers have been assembled. The wire position is measured using X-ray tomography showing an accuracy of $\sim 10-15$ $\mu \mathrm{m}$, well within the specification of $20 \mu \mathrm{m}$. The single tube resolution has been measured to be $\sim 80 \mu \mathrm{m}$.

\subsection{The Thin-Gap Chambers (TGCs)}

The TGCs are similar to MWPCs with a difference that the anode wire pitch $(1.8 \mathrm{~mm})$ is larger than the cathode-anode $(1.4 \mathrm{~mm})$ separation. The anode wire plane is sandwiched by supporting spacers between two cathode planes made of FR4 plates on which graphite is deposited. On the backside of the cathode plane etched copper strips provide capacitive readout orthogonal to the wires. The chamber is operated in saturated mode and uses $\mathrm{CO}_{2}$ n-pentane ( $55 \%-45 \%$ ) mixture. The wire diameter is $50 \mu \mathrm{m}$ and the operating voltage is 3 $\mathrm{kV}$. The TGCs are constructed in doublets or triplets of planes separated by $20 \mathrm{~mm}$ thick paper honeycomb panel which makes a rigid mechanical structure. One thousand doublets or triplets have been manufactured so far [20 $2 \overline{8}]$.

\subsection{The Resistive Plate Chambers (RPCs)}

RPCs are essentially parallel plates chambers: a narrow gas gap of $\sim 2 \mathrm{~mm}$ is formed from two parallel resistive bakelite plates $\left(\sim 10^{10} \Omega . \mathrm{cm}\right)$ separated by insulating spacers. The spacers are glued on the plates at $10 \mathrm{~cm}$ intervals. The internal surfaces are usually coated with a thin uniform layer of linseed oil that is then polymerized. It is thought that because this leads to a smoother surface the intrinsic noise is much reduced. The outside surfaces of the resistive plate are coated with graphite paint and are connected to $\mathrm{HV}(\sim 10 \mathrm{kV})$ and ground respectively, generating a high uniform electric field. The graphite electrodes are separated from capacitive pickup strips by $200 \mu \mathrm{m}$ thick insulating PET films glued on both graphited layers. The signal can be readout using orthogonal strips, enabling 2-D 
position measurement. The chambers have a good time resolution $(\sim$ few ns) and the signal duration is short $(<25 \mathrm{~ns})$.

RPCs can be operated both in the avalanche mode and streamer mode. The avalanche mode offers a higher rate capability and stable timing performance that is independent of counting rate and is the mode suitable for operation at the LHC. In ATLAS the gas mixture used is tetrafluoroethane $\left(\mathrm{C}_{2} \mathrm{H}_{2} \mathrm{~F}_{4}\right)$, a non-flammable and environmentally safe gas, and $\sim$ $3 \%$ iso-butane $\left(\mathrm{C}_{4} \mathrm{H}_{10}\right) ; 0.3 \% \mathrm{SF}_{6}$ may be used to suppress streamer formation. At $\sim$ $10 \mathrm{kV}$ the streamer probablility is about $0.1 \%$ and the rate capability is expected to be up to $1 \mathrm{kHz} / \mathrm{cm}^{2}$.

Considerable attention has recently been paid to RPCs due to the experience of BaBar where the oiled RPCs, run in streamer mode, showed significant ageing. Significant and continued efficiency drop was observed even after increasing the bias voltage. Some of the affected chambers were opened and oil droplets were found instead of a smooth surface. A new oil treatment is now employed: a 'diluted' mixture of $60 \%$ (instead of $40 \%$ ) eptane/linseed oil is used and a single pass (instead of 3) is made resulting in thinner coating $(\Delta \mathrm{t} \sim 10 \mu \mathrm{m}$ instead of $\sim 100 \mu \mathrm{m}$ ). Furthermore smoother bakelite plates are now used. Much effort has also been put into quality control procedures at the gap fabrication plant. It is now believed that these chambers should not age.

A 15-month ageing test has been performed at CERN-X5 GIF irradiation facility. After $0.3 \mathrm{C} / \mathrm{cm}^{2}$, corresponding to 10 ATLAS LHC years, the tested RPC still had a rate capability of $200-300 \mathrm{~Hz} / \mathrm{cm}^{2}[2 \overline{2} \overline{8}]$.

Results were reported at this conference on chambers operated in the streamer mode [2] $\overline{2} \bar{y}]$. Prototypes with 'diluted' oil coating were subjected to 2 months of irradiation in GIF at a rate between $5-150 \mathrm{~Hz} / \mathrm{cm}^{2}$. Ageing was not found to be dramatic and double coating seemed to be more resistant to ageing!

CMS has been developing uncoated RPCs by smoothing the bakelite surfaces before assembly. Although many of the operating specifications can be met one has caused some concern. Un-oiled chambers have a higher intrinsic noise rate: some 10 times that from an oiled chamber. In CMS this causes a high trigger rate that does not allow a sufficient margin for unforeseen problems. As a consequence CMS will also oil the chambers in the barrel region.

A major production run of RPCs, for both CMS and ATLAS, will take place soon with increased emphasis on quality control and monitoring of the oiling step. Initially every tenth chamber will be opened for inspection of the polymerized oiled-surfaces.

Within CMS, R\&D is still being carried out to reduce the intrinsic noise of un-oiled chambers. For example, the intrinsic noise is found to decrease by a factor of three when the graphite coating is offset from the edge of the bakelite plate by $3 \mathrm{~cm}$ instead of the usual $1.5 \mathrm{~cm}$. In the endcap region the neutron-induced noise rate (due to gammas from n-capture reactions) is similar or higher than the intrinsic one and un-oiled chambers in this region would be preferred, as long-term ageing in a high radiation environment is still a concern. 


\section{Electronics}

The subject of the electronics tends to be overlooked in such presentations. In the current large-scale experiments around one-third of the cost of the sub-detectors resides in electronics. In the harsh environment of the LHC the risks to the proper functioning of the detector-system are considerably higher than this cost percentage. All the electronics inside the experimental caverns of LHC pp experiments has to be rad-tolerant whereas those in the high radiation environment (inner tracker, ECAL etc.) have to be radiation-hard. These requirements pose new challenges for HEP experiments. This, together with the development of advanced microelectronics technology has meant that an unusually large fraction of the electronics has had to be custom-designed.

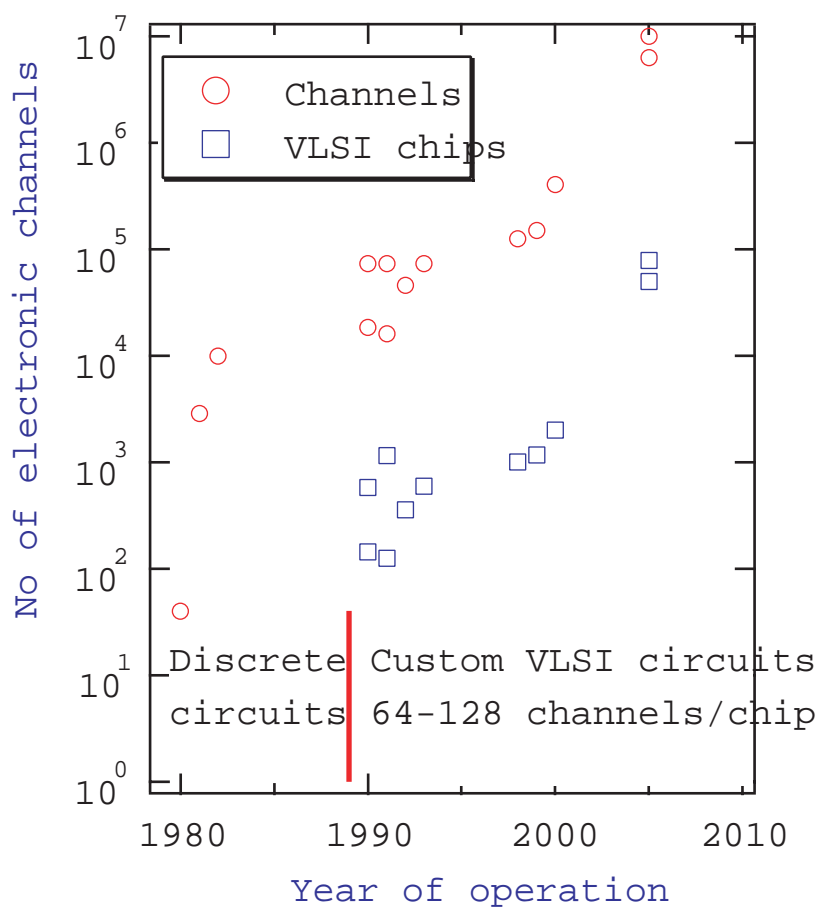

Figure 15: The evolution of the number of electronic channels and chips used in experiments.

The line width found in microelectronics circuits has been decreasing exponentially with time over the last thirty years; it has decreased in commercial circuits from $2 \mu \mathrm{m}$ in 1985 to $0.25 \mu \mathrm{m}$ in 2000 . This has meant that the cost per/channel could be lowered and larger and larger number of channels could be envisaged opening the possibility of building better performing detectors. There has been an almost exponential increase in the number of channels per experiment (Fig. 15) although the experiments themselves have become bigger as well!

In the last decade considerable effort has gone into designing highly performing, low power consuming, low cost/channel and radiation-hard electronics. For most of the last decade electronics designers had worked with foundries traditionally supplying radiation- 
hard electronics to defence and space applications. The up-front costs have been high, and the turn-around times long, hampering rapid development. More recently, as the line width has decreased, deep sub-micron technologies, with some modification, have been found to satisfy the radiation hardness requirements. Most of the R\&D for the LHC pp experiments has been completed and the production phase is being entered.

To illustrate the above mentioned requirements and developments we take, as an example, the electronics chain of the CMS tracker. On the Si detector each microstrip is read out by a charge sensitive amplifier with $\tau \sim 50 \mathrm{~ns}$. The output voltage is sampled at the beam-crossing rate of $40 \mathrm{MHz}$. The samples are stored in an analog pipeline up to the Level-1 trigger latency $(3.2 \mu \mathrm{s})$. Following a trigger a weighted sum of 3 samples is formed in an analog circuit. This confines the signal into single bunch crossing and gives the pulse height. The buffered pulse height data are multiplexed out on optical fibres. The output of the laser is modulated by the pulse height for each strip. The light pulses are transported via $120 \mathrm{~m}$ long optical fibres to the underground control room that is accessible during data taking. The light signal is converted into an electrical signal by Si photodiodes and digitized to give the pulse height. After zero-suppression and formatting the data are stored in local memories ready for access by the DAQ system. Unlike most previous experiments, the functions up to and including light transmission into the optical fibre, are carried out in custom-designed radiation-hard electronics. It should be noted that most of the data of the LHC pp experiments will be transported out on optical fibres. Installation and commissioning of such a large optical cable plant will be a challenge in its own right!

The design of the CMS tracker front-end chip (APV) was almost finalized in 1997 and found to satisfy the radiation-hardness specification. However the vendor decided to carry out the production in a newly set-up foundry and the extreme radiation hardness requirements could no longer be met. As force-majeure CMS decided to investigate a path that looked promising utilizing a foundry that mass-produced chips in $0.25 \mu \mathrm{m}$ CMOS process ('deep-submicron' process). Many radiation-hard circuits for the LHC experiments have been, or are being, converted into this technology. We consider below the reasons

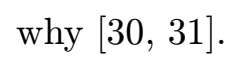

Radiation effects can be divided into two categories: total dose effects and single event

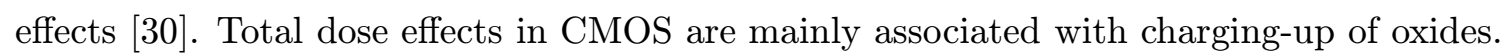
During irradiation electrons and holes are generated in the oxides. While the electrons are evacuated rapidly (within ns) holes accumulate in traps leading to transistor threshold shifts. As the oxides get thinner the charging decreases in proportion to the volume of the oxide. Hence thinner oxides are inherently more radiation hard. As the oxide thickness falls below $10 \mathrm{~nm}$ the reduction in the radiation induced threshold voltage becomes even more pronounced. It is thought that electrons that tunnel into the oxide combine with radiation induced trapped holes. Therefore for deep sub-micron processes (a $0.25 \mu \mathrm{m}$ process has a gate oxide thickness of $5 \mathrm{~nm}$ ) radiation induced threshold voltage shift becomes negligible even at very high radiation doses. However there is still the possibility of leakage paths from drain to source and from one transistor to another that have to be eliminated by

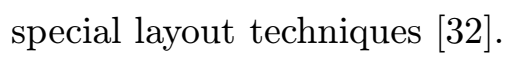

Single event effects are also important especially for the inner trackers at the LHC. 
These are considered in order of severity.

Single event gate rupture (SEGR) is a catastrophic breakdown of transistor gate that occurs when a highly ionizing particle induces an avalanche breakdown of the gate oxide. Fortunately the effect only manifests itself above a critical threshold electric field and should not be an issue for deep sub-micron CMOS circuits.

Single event latch-up (SEL) occurs when a highly ionizing particle deposits enough charge in a small volume of the Si substrate to switch on a parasitic thyristor that causes a very high current to flow between power supplies. If this current is not detected quickly and the power supplies interrupted the chip may be destroyed. One way of avoiding SEL is to use guard rings and substrate contacts extensively in the design. Fortunately these are also the design techniques used to limit total dose effects mentioned above. The 0.25 $\mu \mathrm{m}$ components have been tested to a LET of $89 \mathrm{MeV} \mathrm{cm}^{2} \mathrm{mg}^{-1}$.

Single event upsets (SEU) occur when highly ionizing particle deposits charge near a low capacitance node causing the logical level of the node to switch state. This effect occurs above a threshold LET. The threshold LET tends to decrease for smaller feature sizes and is a real concern for deep sub-micron circuits.

In the 1999 edition of the roadmap of the Semiconductor Industry Association gate lengths are predicted to fall from the present-day $180 \mathrm{~nm}$ to $35 \mathrm{~nm}$ by 2014 accompanied by a fall in the power supply from the present $1.8 \mathrm{~V}$ to $0.6 \mathrm{~V}$. The shrinking of the transistor dimensions should enable continuation of the exponential increase in component density with time. Understanding the limits, and applicability, of such electronics should be a subject of vigorous $\mathrm{R} \& \mathrm{D}$ in our field for the next generation of experiments and for the upgrades of LHC experiments.

The response function of the CMS-APV25 $0.25 \mu \mathrm{m}$ chips is unchanged for doses up to 20 Mrads. These chips have been coupled to final Si modules and have yielded the expected performance: $\mathrm{S} / \mathrm{N}$ of $\sim 15$ in the de-convolution mode. The final chip, with a size of $\sim 7 \times 8 \mathrm{~mm}^{2}$, was received in August 2000. Following these technology developments has resulted in electronics that have lower cost, have lower noise, are made in foundries catering for volume rather than niche markets which has resulted in a faster turnaround from design to working chips.

\section{Future Challenges}

Extreme performance requirements invariably force the pace of detector development. The requirements for the LHC experiments have been a supreme example of this. The linear collider experiments will necessarily push the limits of pixel detectors and high granularity dense calorimetry. Upgrading the LHC to attain luminosities of $10^{35} \mathrm{~cm}^{-2} \mathrm{~s}^{-1}$ is already being discussed. This will require further $R \& D$ especially for the inner tracking detectors (monolithic pixel detectors, macro-pixel detectors, use of defect engineered silicon, Si detectors with novel biasing schemes, Si detectors run at cryogenic temperatures, new sensor materials, engineering issues), for the associated electronics (10-fold increase in number of channels at 10 times lower cost per channel, radiation hardness of electronics at tens of 
$\mathrm{nm}$ feature size), for higher bandwidth optical links for data transport (10 Gbit/s) with rad-hard transmitter end etc.

\section{Conclusions}

It is clear that much progress has been made in detector development in the last decade. This has been driven by the physics goals of the LHC experiments. These experiments have now moved into almost industrial-scale mass production. An important lesson being learnt is the importance of putting enough thought, early enough, into the systems aspects. The new generation of experiments will reap many of the benefits from the $\mathrm{R} \& \mathrm{D}$ that has been necessary for LHC experiments. It is important to acknowledge that improvement of existing techniques and measurements and search for novel ones of are essential in the exploration of uncharted science. It is therefore essential to continue funding of detector (and accelerator) R\&D even at a modest level.

\section{Acknowledgements}

We would like to thank the organizers of this conference for their hospitality and help. We would like to thank all the speakers in the parallel session 13 for illuminating talks and providing beforehand electronic copies of their transparencies. Austin Ball and Peter Sharp are thanked for commenting on the manuscript. Sasha Nikitenko is thanked for help in preparing this manuscript and Guy Martin for drawing some of the figures.

\section{References}

[1] The CMS Electromagnetic Calorimeter Project, Technical Design Report, CERN/LHCC 97-33, Dec. 1997

[2] A. Annenkov et al., to be submitted to Nucl. Instr. and Meth. A and references therein.

[3] M. Doucet, these proceedings.

[4] K. Honscheid, these proceedings.

[5] http://cmsdoc.cern.ch/cms/TDR/MAGNET/magnet.html

[6] C. Damerell et al., IEEE Trans. Nucl. Sci., 33 (1986) 51.

[7] T. Abe, Proc. Workshop on Vertex Detectors (VERTEX 99), Netherlands, June 1999.

[8] M. Winter, these proceedings.

[9] http://cmsdoc.cern.ch/TDR/TRACKER/tracker.html

[10] D. Pitzl et al., Nucl. Instr. And Meth. A311 (1992) 98.

[11] R. Wunstorf et al., Nucl. Instr. And Meth. A315 (1992) 149.

[12] K. Gill et al., Nucl. Instr. And Meth. A322 (1992) 177.

[13] http://cmsdoc.cern.ch/cms/TDR/TRACKER/tracker-addendum.pdf

[14] J. Libby, these proceedings. 
[15] G. Hall, private communication.

[16] A. Oed, Nucl. Instr. And Meth. A263 (1988) 351.

[17] F. Sauli, Nucl. Instr. And Meth. A386 (1997) 531.

[18] R. Bellazzini et al., Nucl. Instr. And Meth. A??? (2000) ???.

[19] G. De Lentdecker, these proceedings.

[20] T. Zeuner, these proceedings.

[21] S. Bachmann et al., Proc. Vienna Conf. on Instrumentation., Feb 2001, Elsevier.

[22] L. Neukermans, these proceedings.

[23] Q. Ingram, these proceedings.

[24] R. Brown, these proceedings.

[25] M. Abolins, these proceedings.

[26] D. Britton, private communication.

[27] M. Calvi, these proceedings.

[28] K. Ishii, these proceedings.

[29] A. Ferretti, these proceedings.

[30] A. Rivetti, 5th Workshop on Electronics for LHC Experiments, CERN 99-09, (1999) 157

[31] M. Campbell et al., Nucl.Instr. and Meth. A 473(2001) 140

[32] P. Jarron et al., Nucl. Phys. B. Proc. Suppl 78 (1999) 625. 